\title{
Editorial
}

\section{The triumph of an idea}

\author{
Uma ideia vencedora
}

Reynaldo André Brandt ${ }^{1}$, Eliova Zukerman ${ }^{1}$

${ }^{1}$ Hospital Israelita Albert Einstein - HIAE, São Paulo (SP), Brazil.

It was a great satisfaction for us the acknowledgment of the publication of einstein's special issue on Neuroscience. This issue constitutes a remarkable development for the Brain Institute (InCe), which is an important part in the story of Hospital Israelita Albert Einstein (HIAE) scientific and educational production.

In 1981 a Training Center in HIAE was established. Since that time, scientific and learning activities were developed for the medical staff of our hospital. Among the Center's activities, there were meetings to discuss neuroscience every week, such meetings counted with the participation of neurologists, neurosurgeons, pediatric neurologists, nurses, physiotherapists, psychologists, and etc. These meetings included scientific paper reviews, formal case presentation and discussions.

Later on, such discussions became morbimortality meetings, the so-called "M\&M meetings", that included debates on complications and adverse results, which aimed the continuing improvement of the neurologic care of our patients. The foundation of the Instituto Israelita de Ensino e Pesquisa Albert Einstein (IIEPAE) in 1998, and the graduate courses in 2001, as well as in the subsequent year, the Research Center, together with the maturing of the neuroscience experts' meetings - circa 20 years now since they have been going on -, served as the basis to establish the InCe on May 23rd, 2003.

From its beginning InCe focused on research and on finding therapeutic applications for neuroscience. The Institute gathers investigators and experts working at HIAE who seek to answer questions for diagnostic, clinical and surgical practice, as well as to address challenges on identifying factors that lead to the appearance of nervous system diseases. Currently, the emphasis of InCe is in identifying genetic factors related to these nervous system diseases using molecular imaging techniques, functional neuroimaging, and in the discovery of therapeutic agents for neurodegenerative diseases (including cerebral implants), in neuromodulation, and in the use of stem cells.

The publications of investigators from InCe have been increasing rapidly and these papers are being published in top medical journals. The Institute partnership with international organizations such as The University of Chicago and the 
Boston University in the United States, the University of Würzburg in Germany, the Weizman Institute of Science in Israel, and the King's College London in England attests the importance of the collaborative work done among neuroscientists and the quality of this research performed together with these institutions. An equal value is given to the partnership with Brazilian institutions like the Universidade de São Paulo with researchers on nanotechnology, the Universidade Federal de São Paulo with investigations on molecular imaging, and the Santa Casa de São Paulo with researches regarding neurochemical and radiopharmacies.

InCe was a pioneer in obtaining the imaging of neurotransmitters and also created a normative basis for functional magnetic resonance imaging in 2005 . In 2008, it obtained the first magnetic resonance imaging in 3T, and in the subsequent year, achieved the first international patent for HIAE. In addition, it was the first institute to apply Single Photon Emission Microtomography in experimental studies.

Nowadays, InCe has more than 40 projects running and its investigators have published over 150 papers in high impact factor medical journals, besides producing master and doctorate thesis.

In the future, like others centers for advanced research, InCe will face many challenges, such as: to maintain the loyalty of its investigators, to attract new physicians to work with science and teaching activities, to find out ways to overcome the bureaucracy to import supplies required for researches, and to acquire the state-of-the-art informatic resources to develop a technological convergence needed to apply new knowledge in healthcare practice, keeping and increasing the technological support as well as funding for the growing of the Institute's activities, etc. Despite being InCe a new institute its short story reveals the nature of a winning entity. 